\title{
FRET-Based Mobile Molecular Nanonetworks
}

\author{
Murat Kuscu Ozgur B. Akan \\ Next-generation and Wireless Communications Laboratory (NWCL) \\ Department of Electrical and Electronics Engineering \\ Koc University, Istanbul, Turkey \\ Email: \{mkuscu, akan\}@ku.edu.tr
}

\begin{abstract}
Nanonetworks refer to a group of nano-sized machines with very basic operational capabilities communicating to each other in order to accomplish more complex tasks such as in-body drug delivery, or chemical defense. Realizing reliable and high-rate communication between these nanomachines is a fundamental problem for the practicality of these nanonetworks. Recently, we have proposed a molecular communication method based on Förster resonance energy transfer (FRET) which is a nonradiative excited state energy transfer phenomenon observed among fluorescent molecules, i.e., fluorophores. We have modeled the FRET-based communication channel considering the fluorophores as single-molecular immobile nanomachines, and shown its reliability at high rates, and practicality at the current stage of nanotechnology. In this study, we focus on network of mobile nanomachines communicating through FRET. We introduce two novel mobile molecular nanonetworks: FRETbased mobile molecular sensor/actor nanonetwork (FRET-MSAN) which is a distributed system of mobile fluorophores acting as sensor or actor node; and FRET-based mobile ad hoc molecular nanonetwork (FRET-MAMNET) which consists of fluorophorebased nanotransmitter, nanoreceivers and nanorelays. We model the single message propagation exploiting the SIR model of epidemics. We derive closed form expressions for the probability of the actor nodes to detect a message generated on the sensor nodes in FRET-MSAN, and for the average detection time of the transmitted message by the nanoreceivers in FRET-MAMNET. We numerically evaluate the performance of these networks in terms of reliability and transmission delay for varying number of nanonodes and varying size of nanomachines, as well as, for several FRET-related parameters.
\end{abstract}

\section{INTRODUCTION}

Nanomachines are envisioned as nanoscale-sized machines with basic operation capabilities such as computing, sensing, actuating. The capabilities of these nanomachines can be improved by enabling communication among them. These nanonetworks are envisaged to accomplish more complex tasks ranging from nuclear defense to treatment of many diseases [1]. Many efforts have been devoted to develop communication methods for future nanonetworks. Electromagnetic and molecular communications are the most promising approaches [2], [3]. Recently, we have proposed a radically different molecular communication method based on the energy transfer between fluorescent molecules, i.e., fluorophores, which provides high communication rates at the molecular level [4].

Fluorophores, e.g., organic dyes, fluorescent proteins, and quantum dots (QDs), are distinct molecules that are able to be excited by optical, electrical, chemical or biological energy, and individually relax to the ground state at a random time by fluorescing, i.e., releasing a single photon with a wavelength at the range of their emission spectrum [5]. An interesting quantum mechanical phenomenon of nonradiative pairwise energy transfer, namely Förster resonance energy transfer (FRET), can occur when at least one spectrally similar acceptor fluorophore is in the close proximity of the excited donor fluorophore. FRET is based on the exchange of excited state energy, i.e., exciton, between an excited donor fluorophore and a groundstate acceptor fluorophore when these molecules get into spectral resonance with each other in a close proximity such as $2-10 \mathrm{~nm}$ [6]. The phenomenon is extensively exploited for biotechnological applications especially by means of spectral ruler or molecular indicators [7], [8].

Based on FRET, recently, we proposed a nanocommunication method between fluorophore-based nanomachines using excitons as information carriers [4]. Employing a donor fluorophore as the nanotransmitter, and an acceptor fluorophore as the nanoreceiver, and using on-off keying (OOK) modulation scheme by encoding 1-bit information into the presence or absence of a single exciton, we information theoretically analyzed the capacity of the channel between them [9]. We also investigated the pulsed excitation scheme in which the information is encoded into an excitonic ns-duration pulse to increase the reliability of the channel [10]. Lastly, with a Monte Carlo approach, we simulated the information transmission through point-to-point channel in a three dimensional aqueous medium comprising a varying concentration of relay fluorophores, and showed that information can be reliably transmitted through $200 \mathrm{~nm}$ distance at a rate over $10 \mathrm{Mbps}$ [11]. However, due to the high degree of randomness, we were not able to give an analytical expression for the probability of successful transmission of information. Furthermore, in the simulations, we assumed that the fluorophores are stationary, and orientation of the fluorophores are constant during the excited state lifetime to ease the computation, therefore, we applied the classical Förster theory which is not valid for diffusing fluorophores with relatively long lifetimes.

In case the fluorophores with sufficiently long excited state lifetimes are used, a donor and acceptor pair with long intermolecular distance at the time of excitation can get into proximity during the lifetime of the donor molecule. Therefore, the probability for an excited donor to get into proximity with more ground-state acceptors during its lifetime increases. As a result of such conditions, the classical Förster theory fails to express the FRET probability for an excited state fluorophore. Fortunately, Stryer et al. derived an expression for the transfer rate of the fluorophores with long lifetimes diffusing in a three dimensional environment [12]. 
In this study, using the expressions derived in [12], [13], we investigate the performance of FRET-based communication between mobile nanonodes for two network scenarios: i) FRETbased mobile molecular sensor/actor network (FRET-MSAN); and ii) FRET-based mobile ad hoc molecular network (FRET$M A M N E T)$. In FRET-MSAN, bioluminescent molecules which are special kind of fluorophores that are excited upon binding a target molecule are considered as the sensor nodes. There are also actuators in the network that can realize a specific task upon receiving an exciton. This deployment can be used for autonomous sensing and actuating tasks at the molecular level. FRET-MAMNET consists of transmitter, relay and receiver nanonodes which are also fluorophores. The single nanotransmitter in the network receives a pulsed excitation signal from an information source and sequentially transmits the excitons to the relay nodes or directly to the nanoreceivers in a probabilistic manner.

In order to model the single message propagation in both networks, we benefit from the SIR model of epidemics [14] which is widely used in modeling mobile ad hoc networks [15]-[17]. In the SIR model, a node can be in three states: susceptible state which corresponds to the ground-state of the nanonodes in FRET-based networks; infected state which is analogous to the excited state of nanonodes; and recovered state which we adapt to our model as the ground-state that comes after the transfer of exciton, i.e., infection, to an actuator or receiver. Similar to the SIR model, we construct the Markov Chain models of both networks. We derive closed form expressions for the probability of a single message to be successfully transferred from the sensor nodes to the actuator nodes in FRET-MSAN, and for the detection time of a single message by one of the nanoreceivers in FRET-MAMNET.

The remainder of this paper is organized as follows. In Section II, we review the FRET theory and rapid-diffusion criterion for mobile fluorophores. In Section III, we present the mathematical models for single message propagation in FRET-MSAN and FRET MAMNET. The results of numerical analysis for performance evaluation are given in Section IV. Finally, the concluding remarks are given in Section V.

\section{THEORY OF FRET}

The theory of FRET between immobile fluorophores is established in Theodor Förster's seminal work [6]. Mainly, there are three conditions for FRET to occur: $i$ ) an excited fluorophore must be in close proximity $(0-10 \mathrm{~nm})$ with at least one ground-state acceptor; $i$ ) the emission spectrum of the donor and the absorption spectrum of the acceptor must overlap; and iii) the relative orientation of dipole moments of the donor and the acceptor fluorophores must not be orthogonal. When these conditions are satisfied, the rate of the energy transfer in terms of the natural fluorescence rate of the donor, i.e., $k_{0}$, is given as

$$
k_{t}=k_{0}\left(\frac{R_{0}}{R}\right)^{6}
$$

where $R$ is the intermolecular distance, and $R_{0}$ is the Förster radius which incorporates the effects of some intrinsic and environmental parameters. $R_{0}$ is given as

$$
R_{0}=\left(8.8 \times 10^{22} \kappa^{2} n^{-4} Q_{D} J\right)^{-\frac{1}{6}}
$$

where $\kappa^{2}$ is the relative orientation factor, $Q_{D}$ is the quantum yield of the donor, $n$ is the refractive index of the medium, and $J$ is the degree of the spectral overlap. $R_{0}$ ranges between $4-10 \mathrm{~nm}$ [5].

In the case of mobile fluorophores, the situation is radically different in the sense that during the excited state lifetime of the donor, the intermolecular distances and the relative orientation of the dipole moments of fluorophores are not constant. Furthermore, the excited donor fluorophore can get in close proximity with a varying number of acceptors if the donor lifetime or the diffusion coefficient of the fluorophores is sufficiently long. Considering that the excitons randomly walk in a random lattice consisting of diffusing fluorophores, giving a closed form expression for the transfer rate requires some assumptions. Stryer et al. postulated the governing rate equations for the energy transfer from a single excited donor to a single ground-state acceptor in a three dimensional environment assuming that the fluorophores are in the rapid-diffusion limit [13]:

$$
k_{r d}=\frac{4 \pi k_{0} R_{0, d-a}^{6}}{3 V a_{d-a}^{-3}}
$$

where $R_{0, d-a}$ is the Förster radius between the donor and the acceptor, and $V$ is the volume of the three dimensional medium. $a_{d-a}$ is the possible closest distance between the centers of the donor and the acceptor. When there are more than one acceptor molecules, the total FRET rate between the donor and the acceptors becomes

$$
k_{t}=k_{r d} N_{a}
$$

where $N_{a}$ is the number of available, i.e., ground-state, acceptor molecules in the environment. The rapid-diffusion criterion is given as

$$
\frac{D \tau_{0}}{s^{2}} \gg 1
$$

where $D$ is the sum of diffusion coefficients of the donor and the acceptor, $\tau_{0}$ is the natural excited state lifetime of the donor, i.e., $\tau_{0}=1 / k_{0}$, and $s$ is the mean intermolecular distance between donor-acceptor fluorophores [13], [18].

The rapid diffusion limit can be achieved by using fluorophores with moderate diffusion coefficients and long lifetimes such as $1-2 \mu s$ [18].

\section{Mathematical Model of FRET-Based Mobile MOLECULAR NANONETWORKS}

In this section, we model the single message propagation both in FRET-MSAN and FRET-MAMNET. We derive closed form expressions for the successful detection probability of a single message in FRET-MSAN, and for the average message detection time in FRET-MAMNET assuming the mobile network nodes, i.e., fluorophores, satisfy the rapid diffusion condition.

We exploit the model of basic epidemic disease spreading to model the information propagation in both networks. In the SIR model of epidemics, there are three possible states for a network node: $i$ ) suspicious state in which the node is susceptible to the illness; $i$ ) infected state in which the node has the illness; and iii) recovered state in which the node is recovered from the illness. Similarly, in FRET-based networks, 


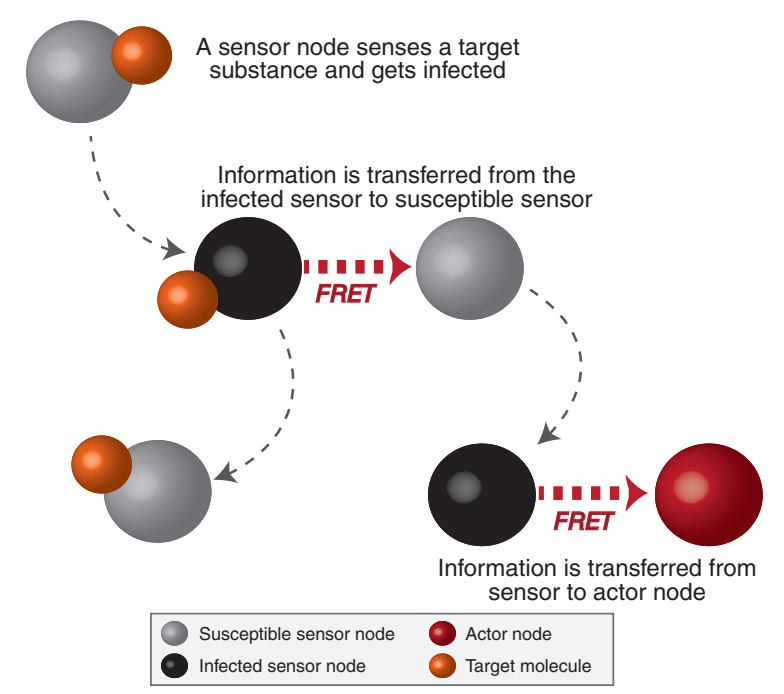

Fig. 1. Information flow in FRET-MSAN.

the nodes except the actors and the receivers can be in the same three states. The susceptible nodes are the molecules that have been never excited or have transferred their exciton to one of the sensor nodes (or relay nodes in FRET-MAMNET), and become susceptible to be excited. The infected nodes are the molecules that have been excited, i.e., infected by an exciton, and cannot be re-excited until they return to the ground state. The recovered nodes are the molecules that have transferred their exciton to one of the actor nodes (or receiver nodes in FRET-MAMNET). The recovered nodes are also susceptible to the infection, however, they do not lead to any ambiguity in the model, because, we model the signal propagation until the first excitation of any actor or receiver nodes. Therefore, the model is valid until one of the network nodes are recovered.

Note that, excited fluorescent molecules can randomly return to the ground state by releasing a photon without transferring the exciton to another molecule at the natural fluorescence rate, i.e., self-relaxation rate [5]. Therefore, in our model, all of the infected nodes are assumed to randomly return to the susceptible state at this rate. Another fundamental difference between our model and the SIR model resulting from the characteristics of FRET is that an infected node gets rid of the infection and returns to the susceptible state when it transfers the infection to another node.

\section{A. Mathematical Model of FRET-MSAN}

Bioluminescent molecules define a class of fluorescent molecules which are excited upon binding a target molecule [5]. Since they do not need a remote excitation source, e.g., optical laser, they are extensively used in biotechnological research as biomolecular sensors optically indicating the presence of a certain kind of molecule [19]. For example, aequorin, a bioluminescent protein, reacts with calcium ions, and relaxes through releasing a photon, thus, it is extensively used to measure $\mathrm{Ca} 2+$ concentration [20].

Fluorescent molecules also find applications in photodynamic therapy (PDT) of cancer as actuators. In QD-based PDT, QDs are excited by optical energy from a remote source and then transfer its exciton to the conjugated photosensitizing

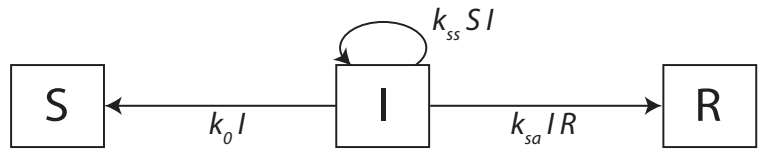

Fig. 2. Markov chain model of FRET-MSAN.

agent which synthesizes a reactive singlet oxygen via energy transfer [21]. The produced singlet oxygen initiates the apoptosis of nearby cancer cells. However, the reactive singlet oxygen is also harmful for normal cells, therefore, the spatial precision of the activation of singlet oxygen is crucial.

Here, we focus on a molecular sensor and actor network, namely FRET-MSAN, composed of mobile bioluminescent sensors and fluorophore-based actors that can collect the information from the sensors and perform an appropriate action upon the environment. The investigated scenario in this section can pave the way for designing autonomous networks of nanomachines which are able to collaboratively sense the presence of tumor cells, and act precisely for the apoptosis of them. The information flow in FRET-MSAN is demonstrated in Fig. 1.

Adapting the SIR model of epidemic disease spreading, we model the signal propagation in FRET-MSAN starting from the generation of excitons on the sensors to the detection by an actor node. We derive a closed form expression for the probability of successful transmission of a one-bit detection message generated on bioluminescent sensors to one of the actor nodes. The model is based on the following assumptions:

- Initially $N_{0}$ number of sensors are in the infected state, i.e., excited state.

- No additional exciton is generated during the message propagation.

- The number of sensor nodes, $N_{s}$, and the number of actor nodes, $N_{a}$, are constant.

- An infected sensor node gets rid of the infection and returns to the susceptible state without transfer of infection at a rate of $k_{0}$. Note that, this is the natural fluorescence rate of the bioluminescent fluorophores used as molecular sensors.

- An infected sensor node transfers the infection to a susceptible sensor node making it infected while returning to the susceptible state with a rate of $k_{t, s s}$.

- An infected sensor node transfers the infection to an actor, and get recovered with a rate of $k_{t, s a}$.

- The transfer of infection is pairwise, i.e., an infected nanonode can transfer its infection to only a single nanonode.

The information transfer rate from an infected sensor node to the susceptible, i.e., available, sensor nodes in the environment is given by

$$
k_{t, s s}=k_{s s} N_{s c}=\frac{4 \pi k_{0} R_{0, s s}^{6}}{3 V a_{s s}^{-3}} N_{s c}
$$

where $k_{s s}$ is the transfer rate between a single pair of infected and susceptible sensor nodes, $N_{s c}$ is the number of susceptible sensor nodes, $R_{0, s s}$ is the Förster radius between 
two sensor nodes, and $a_{s s}$ is the intermolecular distance of closest approach of two sensor nodes. We assume that the bioluminescent sensors are spherical with radius $r_{s}$, therefore, $a_{s s}=2 r_{s}$. Similarly, the rate of the information transfer from an infected sensor node to an actor node is given by

$$
k_{t, s a}=k_{s a} N_{a}=\frac{4 \pi k_{0} R_{0, s a}^{6}}{3 V a_{s a}^{-3}} N_{a}
$$

where $k_{s a}$ is the transfer rate between an infected sensor node and an actor node, $N_{a}$ is the number of actor nodes in the environment, $R_{0, s a}$ is the Förster radius between a sensor node and an actor node, and $a_{s a}$ is the intermolecular distance of closest approach of a sensor node with an actor node. Assuming that the actor nodes are also spherical with radius $r_{a}, a_{s a}=r_{s}+r_{a}$.

Based on the listed assumptions, and following a similar way with the SIR model, we construct the Markov chain model of the single message propagation as demonstrated in Fig. 2. Here, $S(t), I(t), R(t)$ denote the number of susceptible, infected and recovered sensor nodes at time $t$, respectively. From the model we infer that until the first time offloading of the message, the number of infected sensor nodes is governed by the following differential equation:

$$
\frac{d I}{d t}=k_{s s} S(t) I(t)-\left(k_{0} I(t)+k_{s s} S(t) I(t)\right)=-k_{0} I(t)
$$

Assuming that $N_{0}$ number of nanosensors are initially infected, i.e., $I(0)=N_{0}$, (8) can be solved as

$$
I(t)=N_{0} \mathrm{e}^{-k_{0} t}
$$

The cumulative distribution function (CDF) of the delay between the generation of a message on the nanosensor and its transmission to an actor node can be derived from (9). Assuming $\operatorname{Pr}($ event in $[0, t))$ is independent of $\operatorname{Pr}($ event in $[t, t+\epsilon)$ ), we can follow the same derivation in [17], and write the differential equation for $F(t)$ as

$$
\frac{d F}{d t}=k_{t, s a} I(t)(1-F(t))=k_{s a} N_{a} I(t)(1-F(t))
$$

Assuming that an exciton is transferred with a probability of 1 , when an infected sensor node and an actor node make contact, an initial condition for the differential equation (10) can be given by

$$
F_{0}=N_{a} N_{0} \frac{4 \pi\left(\left(r_{a}+2 r_{s}\right)^{3}-r_{a}^{3}\right)}{3 V}
$$

Note that, $F(0)$ is the probability that an initially infected sensor node is adherent with an actor node. Using $F(0),(11)$ can be solved as

$$
F(t)=1-\left(1-F_{0}\right) \mathrm{e}^{\frac{k_{s a} N_{a} N_{0}}{k_{0}}\left(\mathrm{e}^{-k_{0} t}-1\right)}
$$

Using (12), the probability of successful transmission of information from bioluminescent sensors to one of the actor nodes can be given by

$$
\operatorname{Pr}(\text { success })=\lim _{t \rightarrow+\infty} F(t)=1-\left(1-F_{0}\right) \mathrm{e}^{-\frac{k_{s a} N_{a} N_{0}}{k_{0}}}
$$

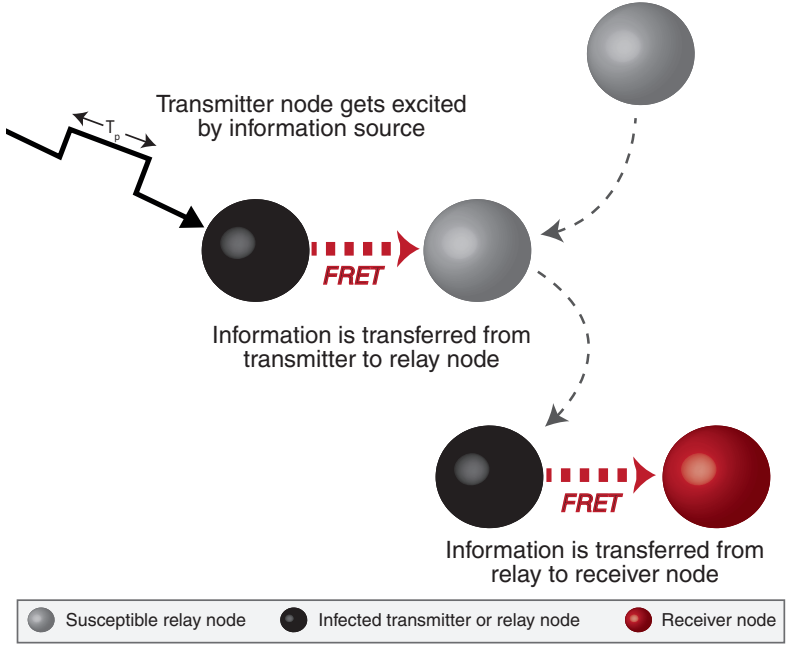

Fig. 3. Information flow in FRET-MAMNET.

\section{B. Mathematical Model of FRET-MAMNET}

In FRET-MAMNET, there are three kinds of fluorophorebased nanonodes randomly dispersed in the same environment: i) nanotransmitter; ii) nanorelays; and iii) nanoreceivers. The single nanotransmitter in the network is continuously excited by an information source, e.g., a remote optical source or a nearby electrical source, with a pulse of duration $T_{p}$. The excited transmitter node generates excitons randomly during $T_{p}$. The relay nodes are not directly excited by an information source. The excitons generated on the nanotransmitter follow random hopping with sequential transfers through the relay nodes, and carry the message from the nanotransmitter to the nanoreceivers. The nanoreceivers are assumed to realize a specific task, e.g., singlet oxygen sensitization, when they receive an exciton. This configuration is similar to the scenario simulated in [11], however, here we assume that the characteristics of the network nodes satisfy the rapiddiffusion limit, therefore, we are able to derive closed form expression for the average detection time of a single message, $T_{d}$. The information flow in FRET-MAMNET is demonstrated in Fig. 3.

We model the single message propagation from the nanotransmitter to a nanoreceiver until an exciton is first received by an receiver node. We again benefit from the SIR model. The model is based on the following assumptions:

- Transmitter and relay nodes are of same type of fluorophores, i.e., they have the same fluorescence rate $\left(k_{0}\right)$, Förster radius $\left(R_{0, h h}\right)$ and molecular radius $\left(r_{h}\right)$.

- There is only a single nanotransmitter that can receive excitation signal from the information source.

- An infected nanonode gets rid of the infection and returns to the susceptible state without transfer of infection at a rate of $k_{0}$.

- An infected nanonode transfers the infection to a susceptible nanonode with a rate of $k_{t, h h}$.

- An infected nanonode transfers the infection to a receiver with a rate of $k_{t, h r}$.

- During the pulse, infection is generated on the transmitter node with a rate of $k_{g}$. 


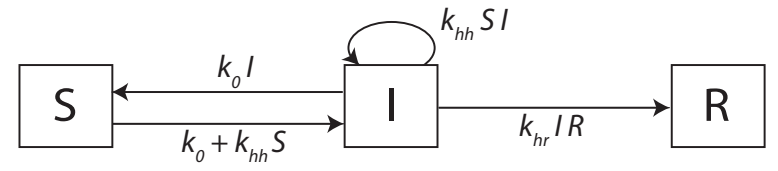

Fig. 4. Markov chain model of FRET-MAMNET.

- $\quad$ Pulse is applied until the message is detected by one of the nanoreceivers, i.e., $\left(T_{p}=T_{d}\right)$.

- The total number of transmitter and relay nodes, $N_{h}$, and the number of receiver nodes, $N_{r}$, are constant.

On the nanotransmitter, when an infection is removed either by self relaxation, i.e., fluorescence, or by transfer to another node, another infection is generated immediately. Therefore, the infection generation rate on the nanotransmitter is given by

$$
k_{g}(t)=k_{0}+k_{t, h h}(t)=k_{0}+k_{h h} S(t)
$$

Note that, $S(t)$ is the number of susceptible nodes, i.e., nodes available for energy transfer, at time $t . k_{h h}$ is the transfer rate between a single transmitter-relay (or relay-relay) pair, that can be expressed as in (3).

The Markov chain model of the single message propagation is given in Fig. 4. In the Markov model, the differential equation for the number of infected nanonodes in the system at time $t$ is expressed by

$$
\begin{aligned}
\frac{d I}{d t} & =k_{0}+k_{h h} S(t)+k_{h h} S(t) I(t)-\left(k_{0} I(t)+k_{h h} S(t) I(t)\right) \\
& =k_{0}+k_{h h}(N-I(t))-k_{0} I(t) \\
& =k_{0}+k_{h h} N-\left(k_{0}+k_{h h}\right) I(t)
\end{aligned}
$$

Considering only a single node, i.e., the transmitter node, is initially infected, the initial condition for $(15)$ is $I(0)=1$. The solution of (15) can be given by

$$
I(t)=K+(1-K) \mathrm{e}^{-\left(k_{0}+k_{h h}\right) t}
$$

where $K=\left(k_{0}+k_{h h} N\right) /\left(k_{0}+k_{h h}\right)$.

Following the same derivation in the first network scenario, the differential equation for the CDF of the average message detection time is expressed as in (10). The initial condition for the CDF, i.e., $F_{0}$ can be given as in (11) with $N_{0}=1$. Using the initial condition, the solution of (10) is given by

$$
F(t)=1-C \mathrm{e}^{\frac{k_{h r} N_{r}(1-K)}{\left.\left(k_{0}+k_{h h}\right) \mathrm{e}^{\left(k_{0}+k\right.} h h\right) t}-K k_{h r} N_{r} t}
$$

with $C=(1-F(0)) \exp \left(k_{h r} N_{r} \frac{k_{h h}(N-1)}{\left(k_{0}+k_{h h}\right)^{2}}\right)$.

Using (17), the average detection time of the message can be expressed by

$$
E\left[T_{d}\right]=\int_{0}^{\infty}(1-F(t)) \mathrm{d} t
$$

which can be numerically computed.

\section{Performance Analysis of FRET-Based Mobile MOLECULAR NANONETWORKS}

In this section, we numerically evaluate the performance of the two network scenario described in Section III. We use the derived expressions of successful detection probability of a single message in FRET-MSAN, and the average detection time in FRET-MAMNET with varying network and node parameters to understand the effect of each parameter on the network performance and gain insight on the feasibility of the FRET-based mobile nanonetworks.

TABLE I. SimUlation Parameters

\begin{tabular}{l|l}
\hline \hline Molecular radii $\left(r_{s}, r_{a}, r_{h}, r_{r}\right)$ & $0.25(\mathrm{~nm})$ \\
\hline Natural fluorescence rate of molecules $\left(k_{0}\right)$ & $10^{6}\left(\mathrm{sec}^{-1}\right)$ \\
\hline Volume $(V)$ & $1\left(\mu \mathrm{m}^{3}\right)$ \\
\hline Förster radii $\left(R_{0, s s}, R_{0, s a}, R_{0, h h}, R_{0, h r}\right)$ & $8(\mathrm{~nm})$ \\
\hline $\begin{array}{l}\text { Number of initially excited sensor nodes in } \\
\text { FRET-MSAN }\left(N_{0}\right)\end{array}$ & 5 \\
\hline $\begin{array}{l}\text { Total number of sensor nodes in FRET-MSAN } \\
\left(N_{s}\right)\end{array}$ & 100 \\
\hline Number of actor nodes in FRET-MSAN $\left(N_{a}\right)$ & 100 \\
\hline $\begin{array}{l}\text { Total number of transmitter and relay nodes in } \\
\text { FRET-MAMNET }\left(N_{h}\right)\end{array}$ & 100 \\
\hline $\begin{array}{l}\text { Number of receiver nodes in FRET-MAMNET } \\
\left(N_{r}\right)\end{array}$ & 100 \\
\hline
\end{tabular}

\section{A. Performance Analysis of FRET-MSAN}

In this section, we present the numerical analysis for the reliability performance of FRET-MSAN using the closed form expression of $\operatorname{Pr}$ (success) derived in Section III. We analyze the single-message transmission probability for varying number of nanoactors and initially infected nanosensors, varying Förster radius between nanosensors and nanoactor, and varying size of nanomachines. Note that, the number of initially susceptible nanosensors, the Förster radius of nanosensornanosensor pair, and the fluorescence rate of the nanonodes do not affect the detection probability. The default values of parameters used in the numerical simulations are presented in Table I.

1) Effect of Number of Actor Nodes: The probability of detection with varying number of nanoactors, $N_{a}$, for different values of Förster radius of nanosensor-nanoactor pair, $R_{0, s a}$, is shown in Fig. 5(a). We observe that the detection probability significantly increases with increasing number of actors, since the chance for an infected nanosensor to be in the communication range of an actor node at any time increases.

2) Effect of Number of Initially Infected Nanosensors: The effect of the number of initially infected nanosensors on the detection probability is shown in Fig. 5(b) with varying number of nanoreceivers. Since we assume that no other external infection occurs, and the infection is only transferred between the network nodes, the number of infected nanosensors is a decreasing function of time. Therefore, the initial number of infection is an important parameter for the detection performance. It is observed that the detection probability significantly increases even with a small increase in the number of initially infected nanosensors. 


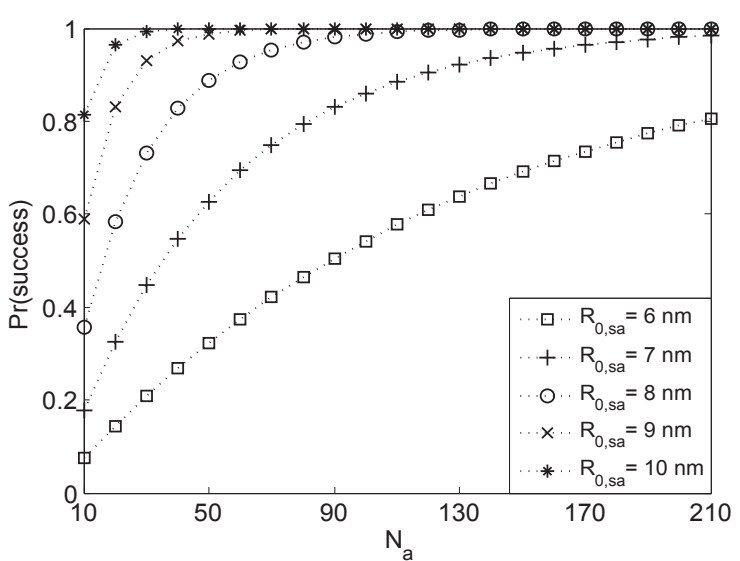

(a) $\operatorname{Pr}$ (success) with varying $N_{a}$ for different $R_{0, s a}$.

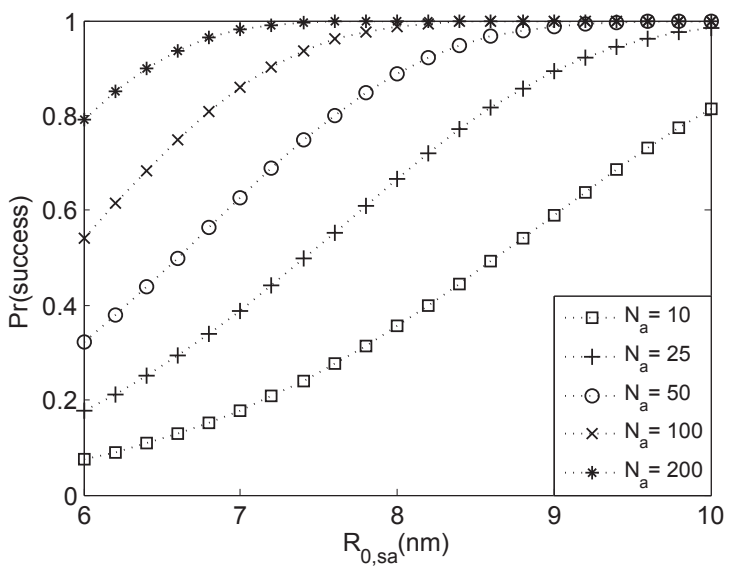

(c) $\operatorname{Pr}$ (success) with varying $R_{0, s a}$ for different $N_{a}$.

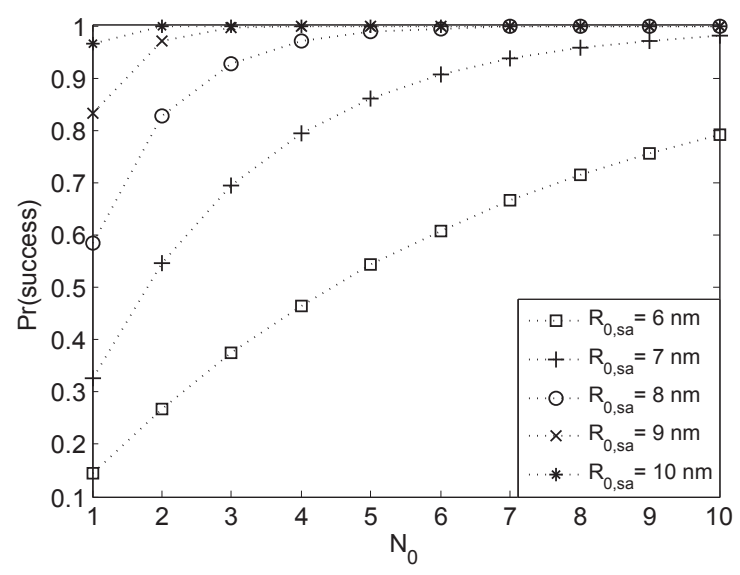

(b) $\operatorname{Pr}$ (success) with varying $N_{0}$ for different $R_{0, s a}$.

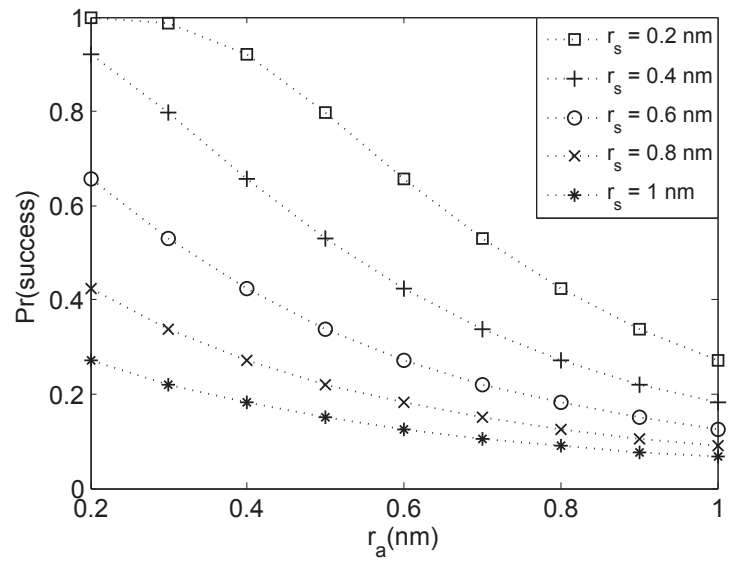

(d) $\operatorname{Pr}\left(\right.$ success) with varying $r_{a}$ for different $r_{s}$.

Fig. 5. Results of numerical analysis for detection probability of single message in FRET-MSAN.

3) Effect of Förster Radius: In Fig. 5(c), Pr(success) with varying $R_{0, s a}$ for different number of receiver nodes is presented. It is shown that as $R_{0, s a}$ increases, the probability of detection is significantly enhanced. In fact, Förster radius is analogous of the communication range of a network node in traditional networks. Therefore, with a large communication range of a nanosensor, more nanoactors as acceptors become available for the energy transfer. As a result, the transfer probability of excitons on each node increases with a decrease in the removal probability of excitons by fluorescence. We also observe that for small values of $R_{0, s i}$, a large number of nanoreceivers are required to successfully detect the message of nanosensors. However, by employing nanosensor-nanoactor pairs with relatively large $R_{0, s a}$, less than 50 receiver nodes will be enough for successful detection.

4) Effect of Size of Nanomachines: The size of nanomachines also significantly affects the successful detection probability, since the extent of the distance of closest approach has a direct effect on the energy transfer rate between nanonodes. The detection probability for varying radii of nanosensors and nanoactors is shown in Fig. 5(d). The selected values for radii are in the range of size of common fluorophores, e.g., fluorescent dyes. We observe that, using small-size nodes significantly increases the detection probability.

\section{B. Performance Analysis of FRET-MAMNET}

In this section, we present the results of numerical simulations to investigate the performance of FRET-MAMNET in terms of average transmission time of a single message, i.e., $T_{d}$. We evaluate the effect of varying number network nodes, varying Förster radii of different pairs, varying lifetime of the nanosensors, and varying size of nanonodes. Note that, different from FRET-MSAN, the number of nanosensors and the excited state lifetime of nanosensors have effect on the performance of FRET-MAMNET. The default values of system parameters used in the simulations are given in Table I.

1) Effect of Number of Receiver Nodes: The number of nanoreceivers significantly affects the detection time as shown in Fig 6(a). This is because, as there are more receiver nodes, the chance for an infected nanonode to enter the proximity of a receiver during the lifetime of its infection increases. Considering point-to-point communication channel with a single nanotransmitter-nanoreceiver pair, i.e., the case of $N_{r}=1$, the detection time is over $25 \mu \mathrm{s}$, even when employing fluorophores with large Förster radius.

2) Effect of Number of Relay Nodes: Fig. 6(b) demonstrates the effect of total number of transmitter and relay nodes on the detection time of a single message with varying $R_{0, h h}$. Note that, there is only a single nanotransmitter, therefore, the 


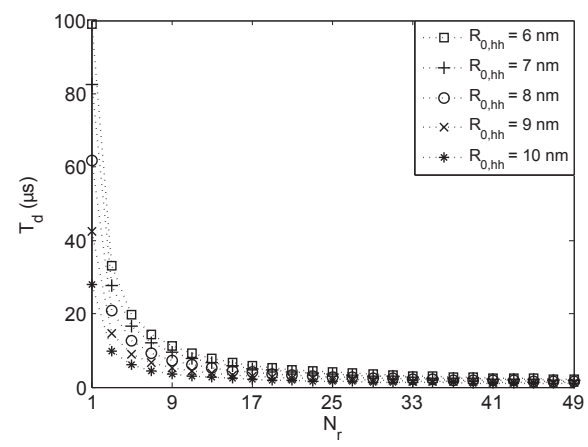

(a) $T_{d}$ with varying $N_{r}$ for different $R_{0, h h}$.

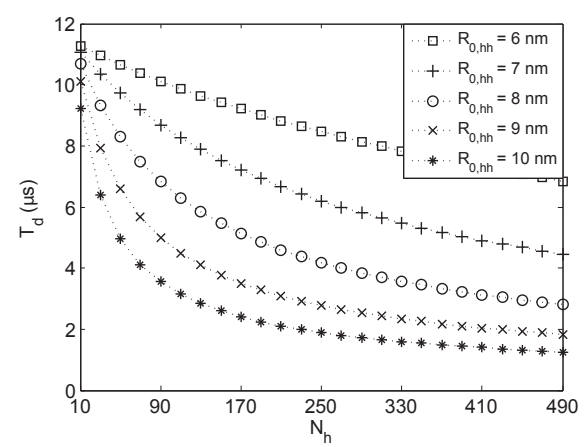

(b) $T_{d}$ with varying $N_{h}$ for different $R_{0, h h}$.

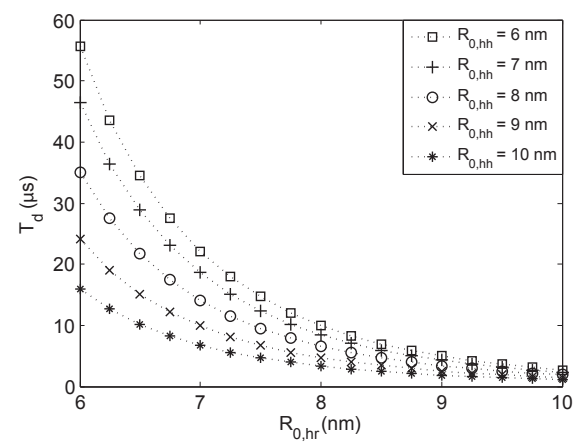

(c) $T_{d}$ with varying $R_{0, h r}$ for different $R_{0, h h}$.

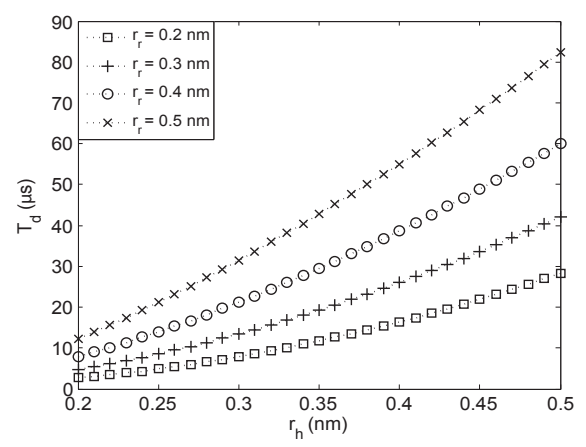

(d) $T_{d}$ with varying $r_{h}$ for different $r_{r}$.

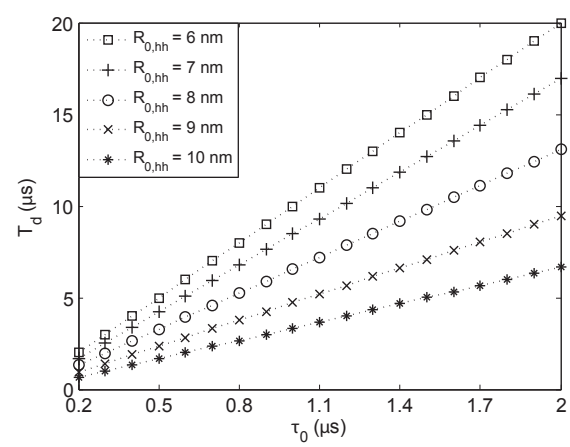

(e) $T_{d}$ with varying $\tau_{0}$ for different $R_{0, h h}$.

Fig. 6. Results of numerical analysis for average detection time of single message in FRET-MAMNET.

number of relay nodes is equal to $N_{h}-1$. It is clearly shown that the detection time significantly decreases with increasing number of nanorelays. Increasing the concentration of the relay nodes increases the number of susceptible nodes in the range of an infected node, thus, increases the transfer rate for each of the nanonode. Therefore, the probability of removal of excitons, i.e., infections, with fluorescence, i.e., self-relaxation, is decreased. Keeping the number of infected nanorelays high increases the probability for a receiver to encounter an infected nanonode in its range, and thus decreases the detection time.

3) Effect of Förster Radius: The detection time significantly decreases with increasing Förster radii of the nanotransmitter, nanorelays and the nanoreceivers as shown in Fig. 6(c). As the Förster radius increases, the effective communication range of a nanonode is improved. Therefore, the number of nodes available for energy transfer in the range of an infected nanonode increases. As a result, the transfer rate for a nanonode increases, as well. This causes the decrease in the detection time.

4) Effect of Size of Nanonodes: The effect of radii of nanonodes is shown in Fig. 6(d). As the radii of nanonodes decrease, the center of nanonodes can get closer, i.e., the distance of closest approach decreases. Therefore, the maximum possible transfer rate between any two nodes increases. Overall, the total exciton transfer rate for each nanonode increases. Higher transfer rates result in lower probability for an exciton to be removed from the network. As a result, the number of excitons randomly hopping through the nanonodes at time $t$ increase, thus, the time required for a receiver to detect an exciton decreases.
5) Effect of Excited State Lifetime of Nanonodes: The effect of excited state lifetime of the nanonodes, $\tau_{0}$, is shown in Fig. 6(e) for different $R_{0, h h}$. It is shown that increasing the lifetime linearly increases the detection time. The lifetime has a significant effect on the transmitter side, such that, it directly affects the exciton generation rate. Lifetime gives a measure of average occupation time of an exciton on a nanonode. When the lifetime is short, the excitons are generated more frequently on the nanotransmitter, therefore, the number of infected nanonodes on the network at time $t$ increases, and the time required for a receiver to encounter an infected nanonode decreases.

\section{CONClusion}

In this paper, based on our recent studies, we introduce FRET-based mobile molecular nanonetworks. We present mathematical models for FRET-based mobile molecular sensor and actor networks (FRET-MSAN) and FRET-based mobile ad hoc molecular networks (FRET-MAMNET) benefiting from the SIR models of epidemic disease spreading. We derive closed form expressions for the single message transmission probability in FRET-MSAN, and for the average detection time of single message in FRET-MAMNET assuming that the network nodes satisfy the rapid-diffusion criterion. Using these expressions, the performance of the networks are evaluated in terms of reliability and delay with varying network and FRET-related parameters. The numerical results reveal that it is possible to achieve reliable communication at high-rates with FRET-based mobile molecular networks.

The deployment of bioluminescent sensor fluorophores and photoactive actor fluorophores makes FRET-MSAN promise 
autonomous applications of target detection and acting at nanoscale such as highly precise PDT applications with singlet oxygen sensitizer fluorophores as actor nodes. FRETMAMNET provides the opportunity of remotely controlling the operation of nanonetworks by an optical information source. This optical link can be made bidirectional if the fluorescence outputs of nanoreceivers are observed by photodetectors, and therefore, it can provide a connection between nanonetworks and larger communication networks, which is currently not possible for other molecular networks proposed so far.

\section{REFERENCES}

[1] I. F. Akyildiz et al., "Nanonetworks: A new communication paradigm," Comput. Netw., vol. 52, no. 12, pp. 2260-2279, 2008.

[2] I. F. Akyildiz and M. J. Jornet, "Electromagnetic wireless nanosensor networks," Nano Commun. Netw., vol. 1, no. 1, pp. 3-19, 2010

[3] T. Nakano et al., "Molecular communication for nanomachines using intercellular calcium signaling," in Proc. of the 5th IEEE Conf. on Nanotechnol., vol. 2, pp. 478481, Nagoya, Japan, July 2005.

[4] M. Kuscu, O. B. Akan, "A nanoscale communication channel with fluorescence resonance energy transfer (FRET)," Proc. 1st IEEE Int. Workshop Molecular Nano Scale Commun./IEEE Conf. Comput. Commun. Workshops, Shanghai, China, 2011, pp. 425-430.

[5] J. R. Lakowicz, Principles of fluorescence spectroscopy, 3rd ed., Baltimore, MD: Springer-Verlag, 2006.

[6] T. Förster, "Zwischenmolekulare energiewanderung und fluoreszenz", Annalen der Physik, vol. 437, pp. 55-75, 1948.

[7] L. Stryer, "Fluorescence Energy Transfer as a Spectroscopic Ruler," Annual Review of Biochemistry, vol. 47, pp. 819-846, 1978.

[8] C. Joo, H. Balci, Y. Ishitsuka, C. Buranachai, T. Ha, "Advances in Single-Molecule Fluorescence Methods for Molecular Biology," Ann. Rev. Biochem., vol. 77, pp. 51-76, 2008.

[9] M. Kuscu, O. B. Akan, "A physical channel model and analysis for nanoscale molecular communications with Förster Resonance Energy Transfer (FRET)," IEEE Trans. Nanotechnol., vol. 11, pp. 200-207, 2012.

[10] M. Kuscu, D. Malak, O. B. Akan, "An information theoretical analysis of broadcast networks and channel routing for FRET-based nanoscale communications," in Proc. 2nd IEEE Int. Workshop Molecular Nano Scale Commun./IEEE Conf. Commun. Workshops, Ottawa, Canada, 2012, pp. 6167-6171.

[11] M. Kuscu, O. B. Akan, "Multi-Step FRET-Based Long-Range Nanoscale Communication Channel," IEEE J. Sel. Areas Commun., to be published.

[12] D. D. Thomas, W. F. Carlsen, L. Stryer, "Fluorescence Energy Transfer in the Rapid-Diffusion Limit," Proc. Natl. Acad. Sci., vol. 75, no. 12, pp. 5746-5750, 1978.

[13] L. Stryer, "Diffusion-Enhanced Fluorescence Energy Transfer," Ann. Rev. Biophys. Bioeng., vol. 11, pp. 203-222, 1982.

[14] M. E. J. Newman, "Spread of epidemic disease on networks," Phys. Rev. E, vol. 66, no. 1, pp. 016128(11), 2002.

[15] A. Khelil, C. Becker, J. Tian, K. Rothermel, "An epidemic model for information diffusion in MANETs," in Proc. 5th ACM International Workshop on Modeling Analysis and Simulation of Wireless and Mobile Systems, Atlanta, Georgia, September 2002.

[16] Z. J. Haas, T. Small, "A New Networking Model for Biological Applications of Ad Hoc Sensor Networks," IEEE/ACM Trans. on Networking, vol. 14, no. 1, pp. 27-40, 2006.

[17] A. Guney, B. Atakan, O. B. Akan, "Mobile Ad Hoc Nanonetworks with Collision-based Molecular Communication," IEEE Trans. Mobile Comput., vol. 11, no. 3, pp. 353-366, 2012.

[18] K. Kinosita, T. Kouyama, A. Ikegami, "Criteria for the Rapid Diffusion Limit of Fluorescence Energy Transfer," J. Chem. Phys., vol. 86, no. 6, pp. 3273-3282, 1987.

[19] C. H. Contag, M. H. Bachmann, "Advances in in vivo bioluminescence imaging of gene expression," Аnnu. Rev. Biomed. Eng., vol. 4, pp. 235260, 2002.
[20] D. G. Allen, J. R. Blinks, "Calcium transients in aequorin-injected frog cardiac muscle," Nature, vol. 273, no. 5663, pp. 509-513, 1978.

[21] A. C. S. Samia, X. Chen, C. Burda, "Semiconductor quantum dots for photodynamic therapy," J. Am. Chem. Soc., vol. 125, no. 51, pp. 15736$15737,2003$. 